\title{
253 Displacement Measurement of Specular Surface by Using Electronic Speckle Pattern Interferometry (ESPI)
}

\author{
Xinbo LI (Yokohama National Univ.) \\ Koichi NISHINO (Yokohama National Univ.) \\ Kahoru TORII (Yokohama National Univ.) \\ Shin-ichi YODA, (NASDA)
}

\begin{abstract}
ESPI is a well-established technique for measuring displacement of rough surface. The possibility of using ESPI on the displacement measurement of specular surface was discussed theoretically in this paper. We described how a modified Michelson interferometer was accommodated to realize this technique. Intensity based fringe analysis was adopted for image processing of speckle grams. Experiment results of using this technique on flat specular surfaces were presented.
\end{abstract}

\section{Keywords: Specular Surface, Displacement Measurement, ESPI}

\section{Introduction}

Electronic speckle pattern interferometry is wellestablished techniques for measuring out-of plane and in pane displacement components, vibrations, slopes, strains and curvatures for a diffusely scattering object [1]. ESPI enable those measurements can be done at the speed of video frame rate (typically 1/30) in the resolution of half $\mu \mathrm{m}$ or even much better. We are trying to develop a technique for measuring free surface wave of liquid bridge whose magnitude is around $1 \sim 2$ $\mu \mathrm{m}$. We can benefit from the advantages of ESPI if the measurement objects of ESPI can be expanded to specularr surfaces. This paper is concerned with the theoretical feasibility and the realization of this extension. Some experiment results on specular surface will be presented too

\section{Theory}

Conventional ESPI technology use speckle pattern as carrier of information, which means this technique will not work on specular object surface [2]. We modified the optical design of classical ESPI system for measuring out-plane displacement by inserting a ground glass before the lens of CCD camera to introduce speckle from original object beam and reference beam.

Fig. 1 shows the principle optical arrangements of three different measurement systems for measuring out-of-plane displacement. Fig. 1 (A) is classical Nichelson interferometer, which works on two nearly identical specular surfaces. Fig. 1 (B) is layout out of conventional ESPI system, which is functional reproduction of (A) while it can work on diffusive surfaces. ESPI technique gets displacement information by comparing the specklegrams captured at different statuses instead of comparing reference surface with object surface at one instant, which means the reference surface needn't to be similar with the object surface Fig. 1 (C) is the modified ESPI system that we are going to present in this paper. This system works on specular surfaces as Fig. 1 (A) does while it uses CCD camera to record specklegrams as Fig. 1 (B) by using the ground glass. The ground glass enable the displacement information can be recorded by low resolution instrument such as CCD camera without worrying about similarity between two specular surfaces.
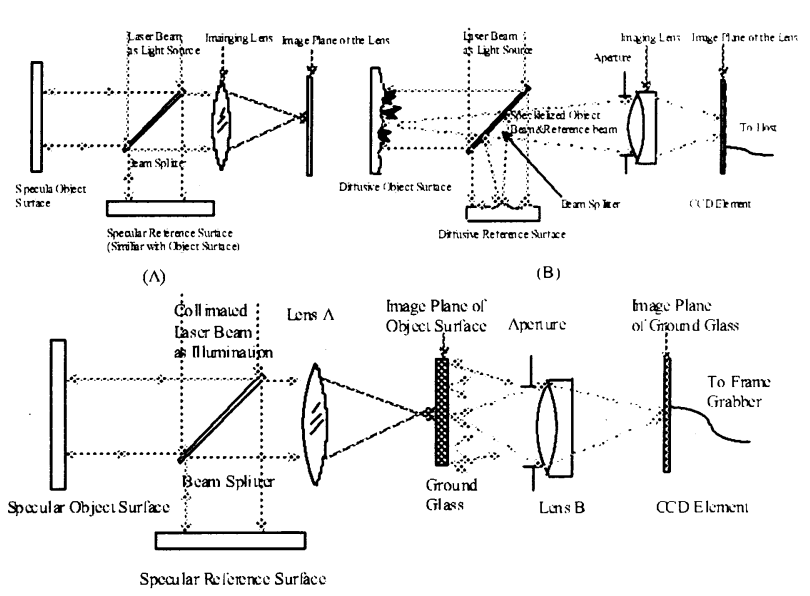

Fig.1 Comparison the optical layouts of: classical Michelson Interferometer (A), ESPI (B), and modified ESPI (C)

In Fig. 1 (C). illumination laser is divided into two beams by beam splitter, illuminates object surface and reference surface separately and be reflected back to 
the beam splitter and then two beams are combined again. The object surface is imaged onto the foreside of the ground glass by the lens A. The ground glass is located on the image plane of object surface since the wave front of the reference surface will not changed during measuring procedure. The CCD camera is focused to the backside of the ground glass to catch speckle patterns.

The wave front of object beam on any point of the CCD camera's image plane is defined by the following:

$$
A_{O}(x, y)=a_{O}(x, y) \exp \left\{i\left[\varphi_{O}(x, y)+\varphi_{G O}(x, y)\right]\right\}
$$

Where: $\varphi_{o}(x, y)$ is the phase part introduced by the surface shape of the object: $\varphi_{G O}(x, y)$ is the phase part introduced by the ground glass during propagation . $a_{o}(x, y)$ is the amplitude.

The wave front function of reference beam can be expressed at the similar way:

$$
A_{R}(x, y)=a_{R}(x, y) \exp \left\{i\left[\varphi_{R}(x, y)+\varphi_{G R}(x, y)\right]\right\}
$$

Where: $a_{R}(x, y), \varphi_{R}(x, y)$ and $\varphi_{i, R}(x, y)$ are corresponding with similar part of the object beam.

The irradiance at any point of CCD element is give $n$ by the following:

$$
\begin{gathered}
I_{F}(x, y)=\left|a_{O}(x, y)\right|^{2}+\left|a_{R}(x, y)\right|^{2}+2\left|a_{O}(x, y)\right| \\
\\
\left|a_{R}(x, y)\right| \cos \left[\varphi_{F}(x, y)\right]
\end{gathered}
$$

Where:

$$
\varphi_{F}(x, y)=\varphi_{O}(x, y)-\varphi_{R}(x, y)+\varphi_{G O}(x, y)-\varphi_{G R}(x, y)
$$

Both $\varphi_{o}(x, y)$ and $\varphi_{R}(x, y)$ are regular distribution since the surfaces are smooth. The interference result of the two surfaces should be regular fringe pattern as any another specular surfaces do if there is no ground glass. But since the $\varphi_{G O}(x, y)$ and $\varphi_{G R}(x, y)$ are randomly distribution, the final image on the CCD element is a kind of speckle pattern, even though sometimes specklized interference fringes due to two specular surfaces can still be distinguished in the image.

If the object surface is displaced or deformed. the direction and the optical path of an individual component of the object beam is changed. However, it is reasonable to assume that the displacement is small enough: the lens A still can image the object surface correctly onto the same resolution area of the ground glass as before [3]. Thus the phase change of the object beam's wave front on the plane of CCD element is determined by change of the initial phase at the foreside of the ground glass, which is a function of the displacement. The propagation procedure in and after the ground glass will not cause obvious additional influence if the displacement is quite small and the Lens A images object surface right on the foreside of the ground glass ${ }^{[4]}$ and that's why Lens $\mathrm{A}$ is needed. The wave front function of object beam will become:

$$
\begin{aligned}
A_{O}(x, y)= & a_{O}(x, y) \exp \left\{i \left[\varphi_{O}(x, y)+\varphi_{G O}(x, y)\right.\right. \\
& +\Delta \varphi(x, y)]\}
\end{aligned}
$$

where: $\Delta \varphi(x, y)$ is introduced by displacement.

The final image captured by CCD camera is given by the following

$$
\begin{aligned}
I_{F}^{\prime}(x, y)= & \left|a_{O}(x, y)\right|^{2}+\left|a_{R}(x, y)\right|^{2}+2\left|a_{O}(x, y)\right| \cdot \\
& \left|a_{R}(x, y)\right| \cos \left[\varphi_{F}(x, y)-\Delta \varphi(x, y)\right]
\end{aligned}
$$

A common method to generate speckle correlation fringes is directly subtracting the image of the displaced surface from that of initial surface state iil pixel by pixel. The subtracted result will be rectified to avoid negative values. The brightness of the subtracterl image is given by the following:

$$
\begin{aligned}
B= & \text { Const } \cdot\left|\sqrt{I_{R} I_{O}}\right| \cdot \mid \sin \left[\varphi_{F}(x, y)+\frac{\Delta \varphi(x, y)}{2}\right] \\
& \sin \frac{\Delta \varphi(x, y)}{2} \mid
\end{aligned}
$$

where: Const is a proportionality constant.

$$
\sin \left[\varphi_{F}(x, y)+\frac{\Delta \varphi(x, y)}{2}\right] \text { represent the speckle }
$$

noise which varies randomly with high frequency across the image.

The brightness will be minimum whenever:

$$
\Delta \varphi=2 n \pi, \quad n=0,1,2, \ldots
$$

While the maximum brightness corresponding with

$$
\Delta \varphi=(2 n+1) \pi, \quad n=0,1,2, \ldots
$$

Since $\Delta \varphi(x, y)$ is a function of the displacement of the object surface, the whole field displacement can be obtained by analysis of the distribution of the fringe pattern. Catherine Wykes proposed a detail analysis of the relationship between phase change and out ${ }^{\circ}$ plane displacement [2], which can be simplified as behind:

$\Delta \varphi(x, y)=\frac{2 \pi}{\lambda}\left(\overline{n_{0}}-\overline{n_{S}}\right) \overline{d(x, y)}$

where: $\overline{n_{O}}$ is unit vector of illuminating laser: $\overline{n_{S}}$ is unit vector of reflection light: $\overline{d(x, y)}$ is displacement distribution on object surface.

If the object surface is a flat specular surface and is illuminated normally by a plane wave front. equation (9) can be simplified to:

$\Delta \varphi(x, y)=\frac{4 \pi}{\lambda} \overline{d(x, y)}$ 


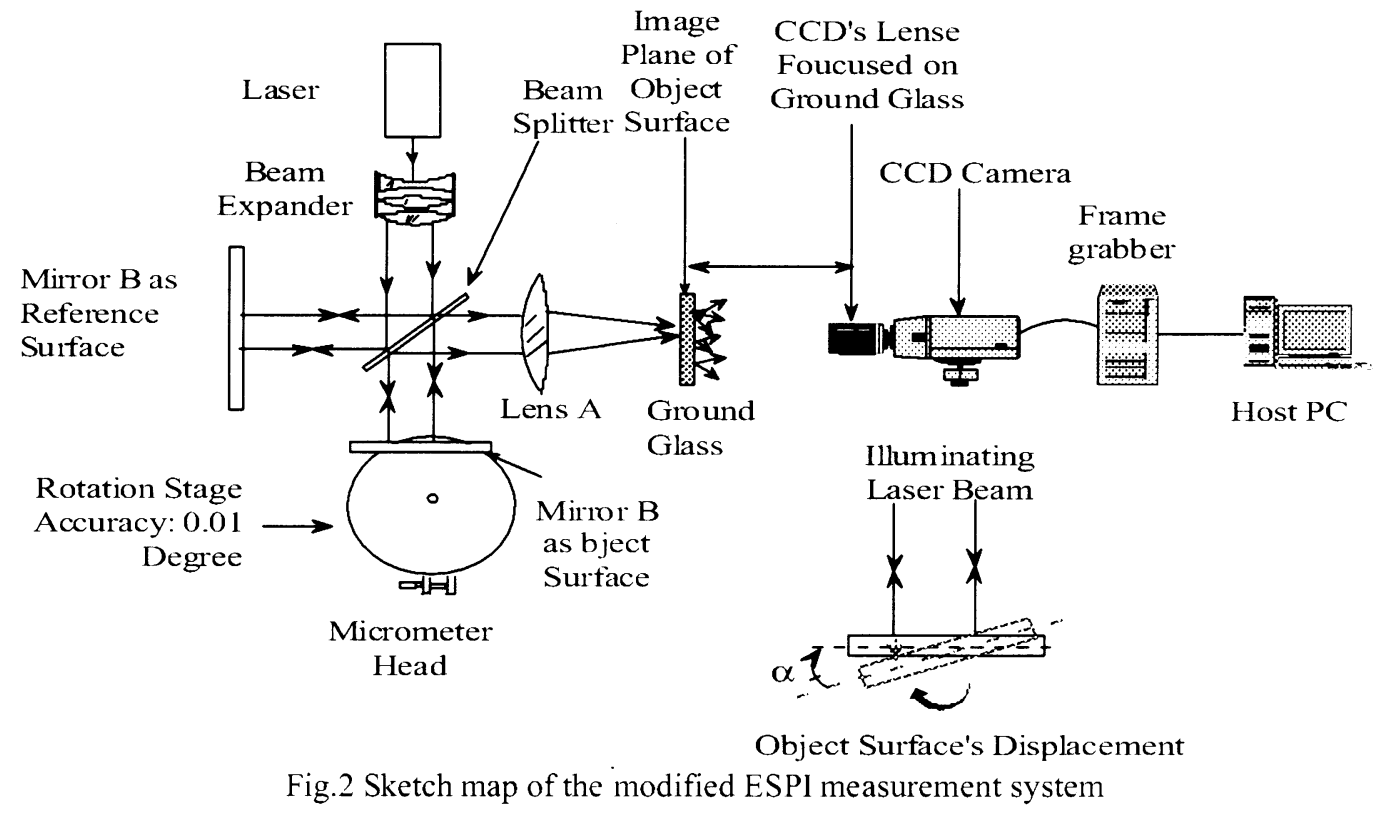

Judging by equation (10), two adjacent maximum brightness values express a half wavelength displacement difference, which is the worst resolution of this system. The resolution can be improved by adopting some interpolation algorithms.

\section{Experiment instrument and results}

Fig.2 is sketch map of the measurement system. Fig. 3 is photo of the modified ESPI measurement system.

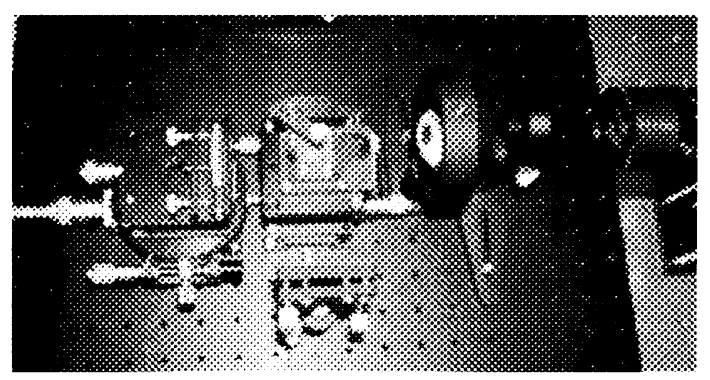

Fig. 3 Photo of measurement system

A flat mirror (Nirror B in Fig. $2 \mu$ ) is used as object surface. It is mounted on a rotation stage. The rotation stage can be rotated in the resolution of $0.012^{\circ}$, which gives $1.44 \mu \mathrm{m}$ displacement on the object surface. Reference surface is another flat mirror (Mirror $A$ in Fig.3). As illumination source a $10^{-} \mathrm{mw}$ laser with 532 $\mathrm{nm}$ wavelength is used. which means the worst resolution of this system is about $260 \mathrm{~nm}$ (half of laser's wavelength). Filters are used to weaken the power of laser. Lens $A$ is an aspheric lens $(\mathrm{f}=39 \mathrm{~mm})$. Distance between Lens A and object surface is $130 \mathrm{~mm}$. The ground glass is $50 \mathrm{~mm}$ far from the back side of Lens $A$. The CCD camera is a SONY AVC-D7 camera. The $2 / 3$ inch CCD array of that camera consists $604 \mathrm{x} 480$ pixels. Lens of the camera is a $55 \mathrm{~mm}, \mathrm{~F} / 2.8$ Kenko telecentric lens. Size of observable area of camera is $7 \times 5.6 \mathrm{~mm}$. Nexus 9000 frame grabber is used to capture series of images during rotating.

Fig. 4 and Fig. 5 are got by subtracting raw images at different status. Fig. 4 is subtracted from two speckle images with $1.44 \mu \mathrm{m}$ displacement. Fig. $\overline{0}$ expresses fringe patterns of $6 \mu \mathrm{m}$ displacement.

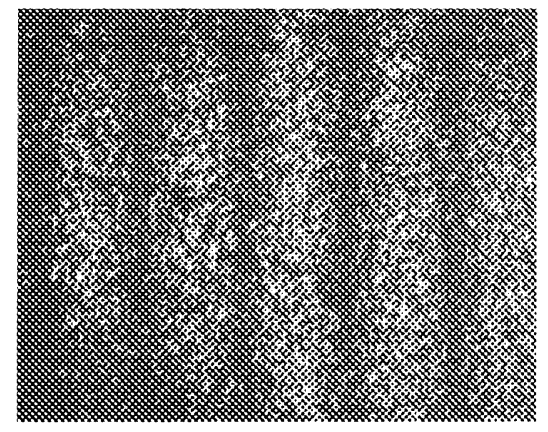

Fig. 4 Fringe pattern of $1.4 \mu \mathrm{m}$ displacement

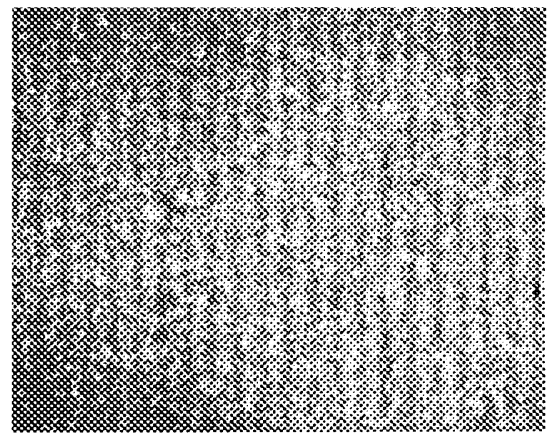

Fig.5 Direct subtracted pattern

When displacement is larger than $6.1 \mathrm{~m}$. fringe pattern that is generated by directly subtraction becomes blurred and undistinguishable due to decor- 
relation of two original images. But if the raw images are subtracted in sequence and the fringe patterns are accumulated, the result agrees well with theoretical

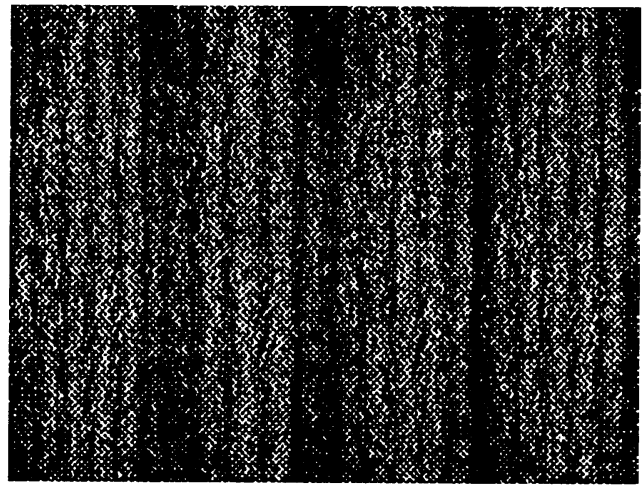

Fig.6 Accumulated pattern of $6 \mu \mathrm{m}$ displacement

estimation, which means lager displacement can be measured by recording and analyzing a series of image during displacing.

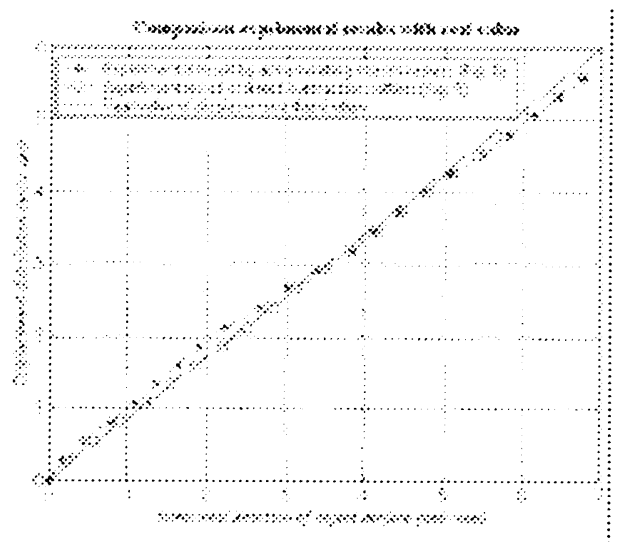

Fig.7 Comparison of experimental results with theoretical values (maximum displacement is $6 \mu$ m)

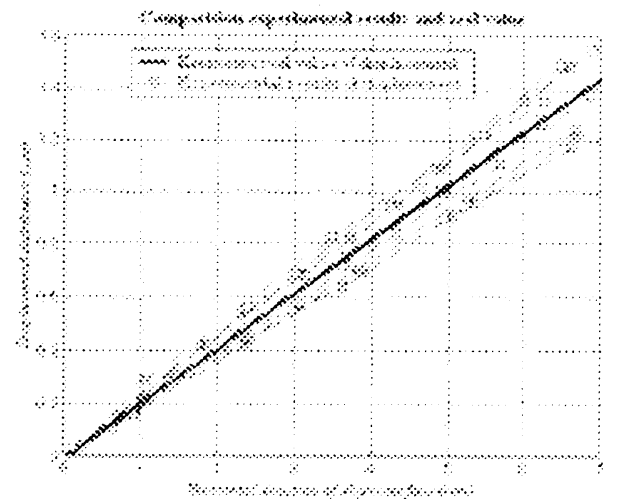

Fig.8 Comparison of experimental results with theoretical values (maximum displacement is 1.44 $\mu \mathrm{m})$
Fig. 7 shows the measurement results and real value of the displacement distribution. Agreement of those results is very well.

Fig. 8 shows comparison of another group of experimental results and "real values" that is estimated to be $1.44 \mu \mathrm{m}$. Displacement in this figure is introduced by rotating only one division of the rotation stage. Random error in rotating due to operator is unavoidable in this situation

\section{Decorrelation of speckle images}

Decorrelation in speckle interferometry introduces errors in the random phases and hence in the interferometric measurement. As described before ESPI method gets fringe by comparing two speckle patterns at different status. The relative phase change between the two speckle patterns is assumed to be introduced only by the displacement or deformation. which is rarely the case. Decorrealtion can be intro. duced by many kinds of resources [1] [t] $[\{j]$. The measurement system presented in this paper suffers from new sources of decorrelation. which is the difference during propagating in ground glass between statuses. Even if the objective lens can image object surface at the exact same position on the ground glass at different status, the change of reflected object beams direction would cause additional phase fluctuation.

\section{Conclusion}

A modification of ESPI technique is presented. This modification enable ESPI technology can be used to measure out-plane displacement of specular surface. Experiment result on flat mirror show well agreement with theoretical estimation.

\section{Reference}

1) Pramod Li. Rastogi. Digital Speckle pattern Interferometry And Related Techniques. John Wiley\&Sons, Ltd. 2001

2) Catherine Wykes. 'Use of electronic speckle pattern interferometry (ESPI) in the measurement of static and dynamic surface displacements , Opt. Eng, Vol.21 (3) 400 . 406

3) Yaozu Song. Wei Zhang. 'Laws of laser speckle morement and their application in flow visualization SPIE Vol 3783, 89·100 (July 1999)

4) Ching Cheng Sun, Wei Chia Su. etc. Three dimensional Random Phase Encoding in Volume Hologram and the Applications'. SPIE Vol. 4110. 139-151 (20100).

5) Charles Joenathan. Pascal Haible and Hans J. Tiziani. Speckle interferometry with temporal phase evaluation: influence of decorrelation. speckle size and nonlinearity of the camera. Applied Optics. Vol. 38. ㅅo. 7. 1169-1178 (1999.)

6) Mathias Lehmann. 'Decorrelation introduced phase errors in phase shifting speckle in terferometry". Applied Optics. Vol. 36. No. 16. 3657 3667 . (1997) 